\title{
Calcitriol and Erythropoietin Protect Against Cardiac Injury Induced by Renal Ischemia-Reperfusion
}

\author{
Mohammad Ghasem Golmohammadi ${ }^{1(\mathbb{D})}$, Ali Shahbazi ${ }^{2}{ }^{(\mathbb{D})}$, Mir Mehdi Chinifroush Asl ${ }^{3}{ }^{(\mathbb{D}}$, Shokofeh \\ Banaei ${ }^{4, *}$ iD \\ 1 Department of Anatomy, School of Medicine, Ardabil University of Medical Sciences, Ardabil, Iran; \\ golmohammadi@gmail.com; \\ 2 General Practitioner, School of Medicine, Ardabil University of Medical Sciences, Ardabil, Iran; shahbazi@ arums.ac.ir \\ 3 Department of Pathology, School of Medicine, Ardabil University of Medical Sciences, Ardabil, Iran; \\ chinifroush@arums.ac.ir; \\ 4 Department of Physiology, School of Medicine, Ardabil University of Medical Sciences, Ardabil, Iran; \\ s.banaei75@gmail.com \\ * Correspondence: s.banaei75@gmail.com;
}

Scopus Author ID 55969784000

Received: 11.04.2020; Revised: 20.05.2020; Accepted: 22.05.2020; Published: 27.05.2020

\begin{abstract}
Cardiac abnormalities and dysfunction are the most important complications after renal ischemia-reperfusion (IR). Thus, investigation and development of effective treatment to decrease cardiac damage induced by renal ischemia are necessary. This study examined the effects of treatment with calcitriol and erythropoietin (EPO) on cardiac injury induced by renal ischemia. Wistar albino rats were unilaterally nephrectomized and subjected to $45 \mathrm{~min}$ of renal pedicle occlusion, followed by $24 \mathrm{~h}$ reperfusion. Calcitriol and EPO were administered before ischemia. After $24 \mathrm{~h}$ reperfusion, blood samples were collected for the determination of biochemical parameters, and kidney and cardiac samples were taken for histological studies. Renal IR increased BUN-Cr levels, lipid profiles, and myocardial injury markers (CK-MB and LDH). Histopathological findings of the IR group confirmed that there were glomerular atrophy and acute tubular necrosis in the renal tissues and lymphocyte infiltration and intercellular edema in the cardiac samples. Treatment with calcitriol and EPO boosted cardiac and renal functions and improved the morphological changes. It seems that calcitriol or EPO administration could protect against the kidney and cardiac damage induced by IR. Also, the combination of calcitriol and EPO may exert more beneficial effects than either agent used alone.
\end{abstract}

Keywords: erythropoietin; ischemia-reperfusion; calcitriol; kidney; heart

(C) 2020 by the authors. This article is an open-access article distributed under the terms and conditions of the Creative Commons Attribution (CC BY) license (https://creativecommons.org/licenses/by/4.0/).

\section{Introduction}

Renal function is impaired by renal ischemia-reperfusion (IR), which is an inevitable problem in clinical situations such as nephrectomy, hydronephrosis, and renal transplantation. There is a deficit in glomerular filtration rate (GFR), some of the hemodynamic parameters are altered, followed by toxins and other unwanted molecules starting to accumulate in the bloodstream. The inflammatory markers, cytokines, and chemokines increase during renal ischemia, consequently compromising the functioning of other organs. The primary renal acute IR injury can culminate with a cardio-renal syndrome with the degrees of electrical dysfunctions and cardiac structural. A considerable peak of serum urea and creatinine $24 \mathrm{~h}$ after reperfusion progressively leads to impairment of renal structure and function. Renal IR 
disturbs some cardiac parameters, and cardiac hypertrophy is developed by renal reperfusion $[1,2]$.

Cardiac arrhythmias are one of the most important complications after kidney ischemia. Kidneys receive $25 \%$ of the cardiac output and highly vulnerable to ischemia. The link between renal IR and cardiac arrhythmias has been credited to electrolyte and water disorders and humoral conditions. Prolonged ventricular repolarization increases the risk of arrhythmias. Remodeling of ventricular repolarization is classically described by disturbances in the regulation of electrolytes such as calcium and potassium and acid-base balance. The production of pro-inflammatory cytokines and reactive oxygen species (ROS) during renal IR can increase the action potential duration of cardio-myocytes and might represent a connection between tissue malfunction, electrical heart abnormalities, and necrosis $[3,4]$.

Calcitriol $(1,25(\mathrm{OH}) 2 \mathrm{D} 3)$ is the biologically active form of vitamin D. It exhibits several physiological activities, including the regulation of calcium homeostasis, immune functions, and antioxidant properties. Calcitriol reduces cell death and necrosis induced by ROS, also, it produces nephron-protective actions. It may be considered a candidate intervention against ischemic invasion. The active form of vitamin $\mathrm{D}$ is produced in the kidney by mitochondria of the proximal tubules. Thus, the levels of calcitriol were decreased after renal IR injury [5,6].

Erythropoietin (EPO) is a hypoxia-induced hematopoietic factor essential for normal erythropoiesis. The important effects of EPO are the reduction in free radicals and inflammatory processes. The recombinant human EPO (rHuEPO) attenuates oxidative stress, the influx of inflammatory cells, and apoptosis. It is revealed that EPO can provide potential protection against IR injury of several organs, including the heart, liver, and lungs $[7,8]$.

Therefore, both calcitriol and EPO have antioxidant and anti-apoptotic effects, and ROS were shown to contribute to the cellular damage induced by IR. The purpose of this study was to investigate the cardioprotective effects of calcitriol and EPO against renal IR damage using biochemical and histological alterations.

\section{Materials and Methods}

\subsection{Animals and surgery.}

For the study, 30 Adult Wistar rats (weighing 200-250 g) were obtained from the experimental animal house of Ardabil Medical Sciences University. All the protocols of the study were approved by the institutional animal ethics committee (ethics code: IR.ARUMS.REC.1398.285). The rats were kept in controlled temperature $\left(21 \pm 2{ }^{\circ} \mathrm{C}\right)$ and humidity $(60 \pm 5 \%)$ environment with free access to water ad libitum and food and under 12-12 hour light-dark cycle.

For induction of the anesthesia, $50 \mathrm{mg} / \mathrm{kg}$ ketamine and $10 \mathrm{mg} / \mathrm{kg}$ xylazine were given by intraperitoneal injection. The animal was placed at the right flank position; after minimal dissection under the last rib, right nephrectomy was performed, and the incision was sutured. Then, the rat was placed at the left flunk position; after minimal dissection under the last rib, the left renal pedicle (artery and vein) was exposed. It was occluded by an atraumatic microvascular clamp for $45 \mathrm{~min}$ to induce ischemia and then subjected to reperfusion for 24 hours. All animals had the right nephrectomy, and the presence of ischemia was visually confirmed by observing the blanching of the kidney. 
The rats were randomly divided into five groups of six rats in each group. The control group $(\mathrm{N}=6)$ underwent the only nephrectomy without occlusion. The other groups were as follows:

Vehicle + IR group; Calcitriol + IR group; EPO + IR group; Calcitriol +EPO+ IR group

Erythropoietin (Neorecormom, Roche, Mannheim, Germany) was administered as a $1000 \mathrm{U} / \mathrm{kg}$ single dose IP, $30 \mathrm{~min}$ before ischemia [9]. Calcitriol used in the present study was obtained from Sigma-Aldrich (USA) and was dissolved in ethanol, diluted with saline, and adjusted to a final concentration of $10 \mathrm{mg} / \mathrm{kg}$ [10]. Calcitriol was injected intraperitoneally, before ischemia, an equal amount of saline supplemented with the required volume of ethanol was used as the vehicle [5].

\subsection{Biochemical measurements.}

Blood samples, cardiac and left kidney tissues were collected after twenty-four hours of reperfusion in each group. The blood samples were centrifuged at approximately 3000rmp for $10 \mathrm{~min}$. The blood urea nitrogen $(\mathrm{BUN})$ and creatinine $(\mathrm{Cr})$ levels to assess the renal function and lipid profile including cholesterol, triglyceride, low-density lipoprotein (LDL) and high-density lipoprotein (HDL) levels in the serum were measured, using the Auto-analyser (Alycon 300 USA) with original kits. The myocardial injury can be diagnosed by measurement of certain enzymes (CK-MB and LDH) released into the blood from the damaged heart muscle, the activities of the lactate dehydrogenase (LDH) and creatine kinase-MB (CK-MB) enzymes were assayed using Randox assay kits [11].

\subsection{Histopathological analysis.}

The heart and renal tissue samples of the experimental animals were sectioned and fixed in $10 \%$ buffered- formalin solution, and then the sections were embedded in paraffin. Sections of $5 \mu \mathrm{m}$ were taken, stained with hematoxylin and eosin (H\&E), and evaluated under a light microscope in a blinded manner by the pathologist. Histological changes were scored on a 4point scale: (-) none, (+) mild, (++) moderate, and (+++) severe damage [12].

\subsection{Statistical analysis.}

The experimental data were expressed as mean \pm standard deviation (SD), using the one-way analysis of variance (ANOVA) followed by a post hoc complimentary test with SPSS 19.0 software. Statistical significance was accepted at $\mathrm{P}<0.05$.

\section{Results and Discussion}

The effect of calcitriol and erythropoietin on the renal ischemia-reperfusion injury was investigated in $45 \mathrm{~min}$ of renal ischemia followed by 24-hour reperfusion. Biochemical analysis results are outlined in Tables 1 and 2. Also, the results of the histological evaluation are shown in Tables 3 and 4.

\subsection{Effects of ischemia-reperfusion.}

The BUN-Cr levels were significantly higher in the IR group compared with those from the control group ( $\mathrm{P}<0.001$, Table 2$)$. The serum levels of cholesterol, triglyceride, and LDL 
in the IR group were higher than those of the control group. Also, in the IR group, the HDL level was lower than that of the control group (Table 1). The enzymes of LDH and CK-MB were elevated in the IR group in comparison to the control group, in which the elevation of these enzymes may result from the damaged heart muscle induced by renal ischemia (Table 2).

Histological examination of the kidneys and heart showed that there were no histological changes in the control group (Figures 1A, 2A). In the IR group, acute tubular necrosis (ATN) was very obvious. There were cell detachment, glomerular atrophy, congestion, and mononuclear cell infiltration following renal IR when compared with the control group (Figure 1B). Also, in the IR group, the typical histological organization of the cardiac tissues was disrupted. There were wavy fibers, intercellular edema, together with an increase in infiltration, and signs of congestion following IR when compared with the control group (Figure 2B).

\subsection{Effects of calcitriol on renal ischemia-reperfusion.}

Serum BUN-Cr levels in the calcitriol + IR group were lower than those of the IR group (Table 2). Serum cholesterol, triglyceride, and LDL levels were significantly decreased in the calcitriol + IR group in comparison to the IR group. Also, the levels of HDL were declined in the group treated with calcitriol (Table 1). The study showed that the serum levels of both LDH and CK-MB were noteworthy declined in the group treated with calcitriol when compared with the IR group (Table 2).

The treatment with calcitriol resulted in a marked reduction of histopathological alterations induced by ischemia-reperfusion in renal and heart tissues. Of course, the mild degree of neutrophil infiltration and intercellular edema were observed in cardiomyocytes in the treated group with calcitriol (Figures 1C, 2C).

\subsection{Effects of erythropoietin on renal ischemia-reperfusion.}

Serum BUN-Cr levels in the EPO+IR group were lower than those of the IR group (Table 2). The serum levels of cholesterol, triglyceride, and LDL were considerably decreased in the group treated with EPO when compared with the IR group, whereas HDL level was elevated by the treatment with EPO (Table 1). Also, the levels of CK-MB and LDH were noticeably reduced in the EPO+IR group in comparison to the IR group (Table 2).

In the group treated with EPO, there were acute tubular necrosis, congestion, and mononuclear cell infiltration in renal tissue. Also, extravasation of RBC and lymphocyte infiltration were observed in the cardiac tissue (Figures 1D, 2D).

\subsection{Effects of calcitriol and erythropoietin on renal ischemia-reperfusion.}

In the calcitriol +EPO+IR group, the serum level of BUN-Cr was lower than that in the IR group (Table 2). The serum levels of cholesterol, triglyceride, and LDL were considerably reduced in the group treated with calcitriol and EPO when compared with the IR group. In contrast, HDL level was increased by the administration of both calcitriol and EPO (Table1). The study indicated that the serum levels of both CK-MB and LDH were noticeably reduced in the calcitriol + EPO group in comparison to the IR group (Table 2).

Calcitriol and EPO co-treatment severely attenuated the histopathological changes, nearly the typical structure of renal and cardiac tissues were preserved by calcitriol and EPO 
pretreatment. Thus, combination therapy appears to have considerable improvement in ischemia-reperfusion injury (Figures 1E, 2E).

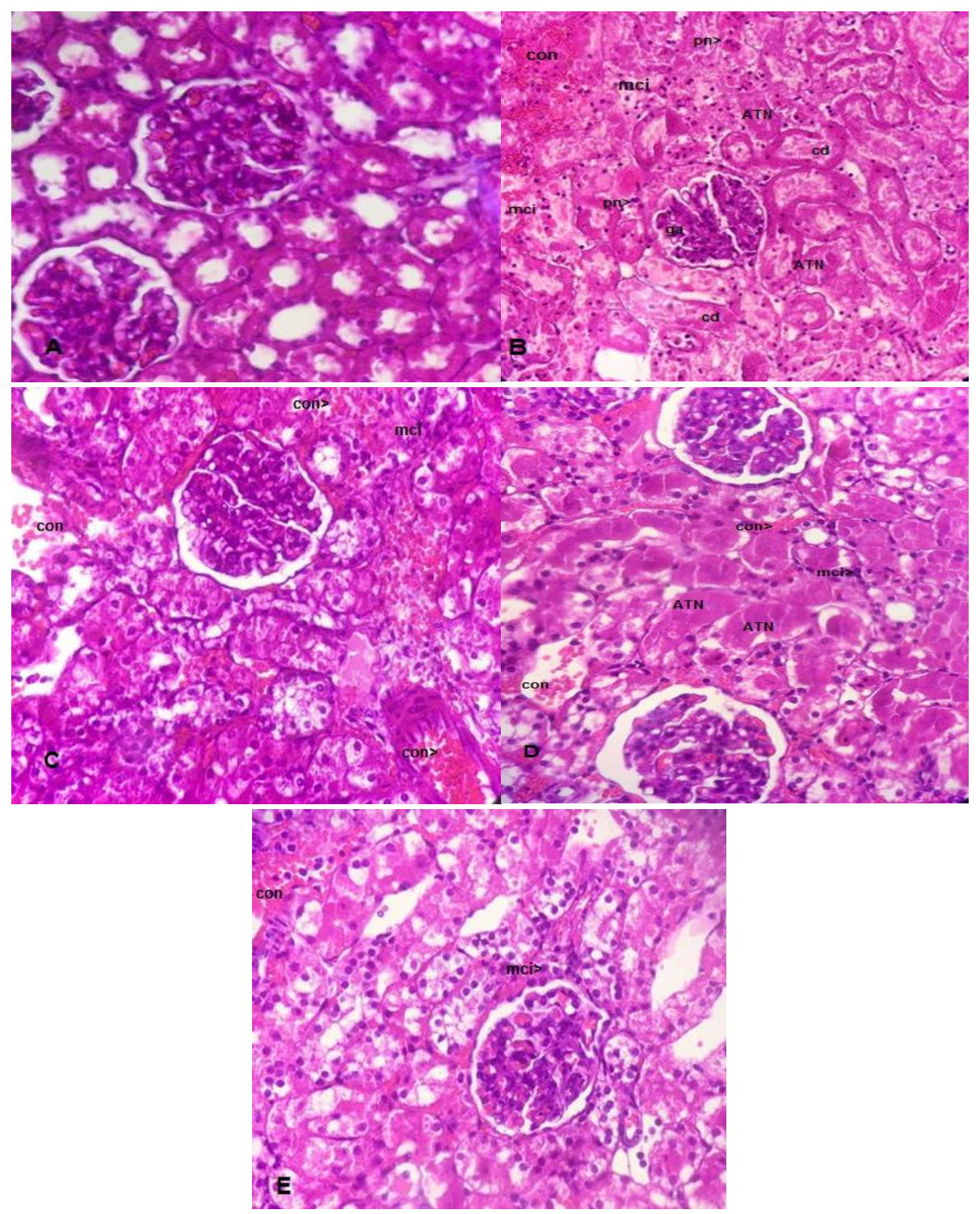

Figure 1. Histopathological evaluation of rat kidneys after $45 \mathrm{~min}$ of ischemia followed by $24 \mathrm{~h}$ of reperfusion. Kidney sections are stained by hematoxylin and eosin (H\&E). (A) The typical renal tissue structure in the control group. Healthy appearance of glomerular and tubular cells $(40 \times \mathrm{HE})$. (B) Cell detachment $(\mathrm{cd})$, acute tubular necrosis (ATN), congestion (con), glomerular atrophy (ga), mononuclear cell infiltration (mci), and pyknotic nuclei (pn) in the IR group $(40 \times \mathrm{HE})$. (C) The lesser degree of mononuclear cell infiltration (mci) and congestion (con) in the calcitriol group $(40 \times \mathrm{HE})$. (D) The degree of acute tubular necrosis (ATN), congestion (con), mononuclear cell infiltration (mci) in the EPO group $(40 \times \mathrm{HE})$. (E) The lesser degree of mononuclear cell infiltration (mci) and congestion (con) in the calcitriol + EPO group $(40 \times \mathrm{HE})$.

Table 1. Biochemical measurements after $24 \mathrm{~h}$ of reperfusion.

\begin{tabular}{l|c|c|l|l|l} 
& \multicolumn{1}{|c|}{ Control } & IR & \multicolumn{1}{c}{ Calcitriol +IR } & EPO+IR & $\begin{array}{c}\text { Calcitriol } \\
+ \text { EPO+IR }\end{array}$ \\
\hline Cholesterol(mg/dl) & $75.16 \pm 9.53$ & $78.88 \pm 9.18$ & $67.78 \pm 14.32$ & $72.46 \pm 17.65$ & $72.50 \pm 4.83$ \\
\hline Triglyceride(mg/dl) & $40.23 \pm 5.93$ & $46.66 \pm 7.28$ & $35.13 \pm 12.70^{\mathrm{a}}$ & $35.80 \pm 4.80$ & $38.43 \pm 6.20$ \\
\hline LDL(mg/dl) & $12.16 \pm 5.07$ & $32.16 \pm 8.99^{\mathrm{b}}$ & $23.66 \pm 8.73^{\mathrm{a}}$ & $28.50 \pm 5.01$ & $26.16 \pm 5.34$ \\
\hline HDL(mg/dl) & $46.83 \pm 4.99$ & $43.06 \pm 3.26$ & $39.76 \pm 8.13$ & $47.36 \pm 22.64$ & $47.21 \pm 8.28$
\end{tabular}

a Significantly decreased when compared with IR group, $\mathrm{P}<0.05$.

b Significantly increased when compared with the control group, $\mathrm{P}<0.05$.

LDL; low-density lipoprotein, HDL; high-density lipoprotein. 


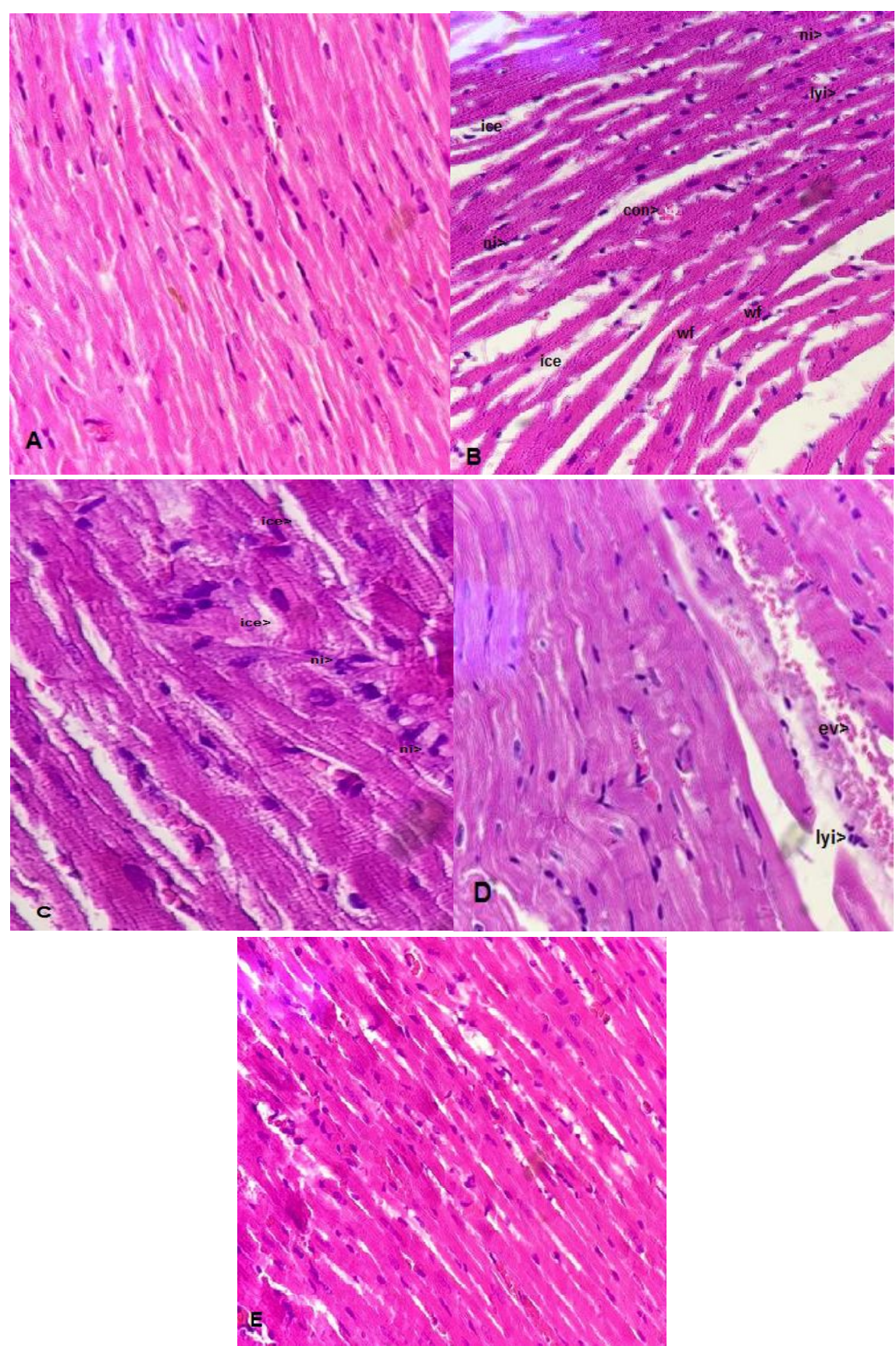

Figure 2. Histopathological evaluation of cardiomyocytes after $45 \mathrm{~min}$ of ischemia followed by $24 \mathrm{~h}$ of reperfusion. Cardiac sections are stained by hematoxylin and eosin (H\&E). (A) The typical myocardial structure in the control group $(40 \times \mathrm{HE})$. (B) Lymphocyte infiltration (lyi), wavy fibers (wf), intercellular edema (ice), neutrophil infiltration (ni), and congestion (con) in the IR group $(40 \times \mathrm{HE})$. (C) The mild degree of intercellular edema (ice) and neutrophil infiltration (ni) in the calcitriol group (40 $\times \mathrm{HE}$ ). (D) Extravasation (ev) and lymphocyte infiltration (lyi) in the EPO group $(40 \times \mathrm{HE})$. (E) The standard and healthy appearance of cardiomyocytes in the calcitriol $+\mathrm{EPO}$ group $(40 \times \mathrm{HE})$.

Table 2. Biochemical measurements after $24 \mathrm{~h}$ of reperfusion.

\begin{tabular}{|c|c|c|c|c|c|}
\hline & Control & IR & Calcitriol +IR & EPO+IR & $\begin{array}{r}\text { Calcitriol } \\
+\mathrm{EPO}+\mathrm{IR} \\
\end{array}$ \\
\hline BUN(mg/dl) & $54.66 \pm 8.61$ & $139.21 \pm 96.54^{\mathrm{a}}$ & $127.51 \pm 19.35$ & $119.25 \pm 40.70$ & $84.93 \pm 12.43$ \\
\hline $\mathrm{Cr}(\mathrm{mg} / \mathrm{dl})$ & $0.68 \pm 0.06$ & $0.96 \pm 0.49$ & $0.83 \pm 0.43$ & $0.90 \pm 0.19$ & $0.81 \pm 0.12$ \\
\hline CK-MB(U/L) & $592.00 \pm 173.67$ & $632.50 \pm 186.68$ & $513.98 \pm 199.58$ & $496.61 \pm 72.31$ & $478.58 \pm 66.34$ \\
\hline LDH (U/L) & $670.00 \pm 33.33$ & $723.16 \pm 217.90$ & $673.66 \pm 379.78$ & $645.50 \pm 213.06$ & $631.83 \pm 109.39$ \\
\hline
\end{tabular}

a Significantly increased when compared with the control group, $\mathrm{P}<0.05$.

BUN; Blood urea nitrogen, $\mathrm{Cr}$; Creatinine, $\mathrm{CK}-\mathrm{Mb}$; creatine kinase-MB, LDH; lactate dehydrogenase. 
Table 3. Tubular and Glomerular changes in the kidney after $24 \mathrm{~h}$ reperfusion (H\&E).

\begin{tabular}{l|l|l|l|l|l|l} 
Groups & $\begin{array}{l}\text { Glomerular } \\
\text { atrophy }\end{array}$ & $\begin{array}{l}\text { Cell } \\
\text { detachment }\end{array}$ & $\begin{array}{l}\text { Acute } \\
\text { tubular } \\
\text { necrosis }\end{array}$ & $\begin{array}{l}\text { Mononuclear } \\
\text { cell infiltration }\end{array}$ & Congestion & $\begin{array}{l}\text { Pyknotic } \\
\text { nuclei }\end{array}$ \\
\hline Control & - & - & - & - & - & - \\
\hline IR & +++ & +++ & +++ & +++ & +++ & +++ \\
\hline Calcitriol & - & - & - & + & ++ & - \\
\hline EPO & - & - & ++ & + & + & - \\
\hline Calcitriol+EPO & - & - & - & + & + & -
\end{tabular}

A minimum of 10 fields for each kidney slide was examined and assigned for the severity of changes using scores on a scale of: $(-)$ none, $(+)$ mild, $(++)$ moderate, and $(+++)$ severe damage. $(\mathrm{n}=6$ for each group)

Table 4. Pathological changes in the cardiomyocytes after $24 \mathrm{~h}$ reperfusion $(\mathrm{H} \& \mathrm{E})$.

\begin{tabular}{l|l|l|l|l|l} 
Groups & $\begin{array}{l}\text { Intercellular } \\
\text { edema }\end{array}$ & $\begin{array}{l}\text { Wavy } \\
\text { fibers }\end{array}$ & extravasation & $\begin{array}{l}\text { Lymphocyte } \\
\text { infiltration }\end{array}$ & Congestion \\
\hline Control & - & - & - & - & - \\
\hline IR & +++ & +++ & ++ & +++ & ++ \\
\hline Calcitriol & ++ & - & - & ++ & - \\
\hline EPO & - & - & ++ & + & - \\
\hline Calcitriol+EPO & - & - & - & + & -
\end{tabular}

A minimum of 10 fields for each cardiomyocyte slide was examined and assigned for the severity of changes using scores on a scale of: $(-)$ none, $(+)$ mild, $(++)$ moderate, and $(+++)$ severe damage. $(\mathrm{n}=6$ for each group)

\subsection{Discussion.}

Acute kidney injury (AKI) induced by ischemia-reperfusion injury is a major cause of mortality among patients during cardio-pulmonary bypass surgery, especially renal transplantation. The prognosis of patients with AKI-induced dysfunction of remote organs, such as the brain, lungs, heart, and liver, remains poor in the clinical setting. The heart is one of the reciprocally triggered organs damaged by systemic injury induced by renal ischemia. AKI has been reported to result in increased levels of oxidative stress, apoptosis and pro-inflammatory cytokines in the heart, which may be associated with considerable morbidity. Therefore, investigation and development of effective adjuvant treatment to decrease cardiac damage from AKI are necessary [13].

In our study, renal IR injury resulted in both tubular and glomerular dysfunction [14]. IR significantly elevated the levels of BUN-Cr, suggesting an impaired glomerular filtration rate, which was decreased by calcitriol or erythropoietin treatment. It is reported that the pretreatment with EPO prior to ischemia reduced the renal dysfunction induced by IR damage [15]. There is recent evidence that calcitriol retards deterioration of kidney function and structure and regulates glomerular filtration [16]. Also, we found that the serum levels of BUN$\mathrm{Cr}$ decreased by the combination of two agents (calcitriol + EPO), which shows that calcitriol + EPO improves the recovery of kidney function.

The present study showed that calcitriol significantly reduced lipid profile in comparison to the ischemia-reperfusion group. Several studies indicated that calcitriol levels were inversely correlated with total cholesterol, triglyceride, and LDL levels. In a study by Bonakdaran et al. [17], supplementation with $0.5 \mu \mathrm{g}$ calcitriol versus placebo in renal failure, patients on hemodialysis lowered the serum total cholesterol and triglyceride. Two mechanisms might be involved in calcitriol mediated reduction in serum triglycerides: 1 . Calcitriol elevates intestinal calcium absorption. This calcium could then decrease the serum triglyceride by decreasing hepatic triglyceride formation and secretion; 2. Via a suppressive effect of calcitriol on serum parathyroid hormone (PTH) concentrations. A decrease in serum PTH may reduce the serum triglyceride via elevated peripheral removal [18]. 
On the other hand, in this study, EPO decreased lipid profile when compared with the IR group. Tuzcu et al. [19] who demonstrated that hemodialysis (HD) patients treated with rHuEPO are insulin sensitive when compared with HD patients not treated with rHuEPO and the treatment with rHuEPO in these patients is an efficient pathway to increase the hematocrit value, to reverse cardiovascular problems, and to improve insulin sensitivity. A decrease in serum lipid profile after EPO administration may be related to an improved response to insulin and hypoglycemic and hypolipidemic actions of insulin. Thus, an improvement in total cholesterol and triglycerides following EPO therapy can be reduced cardiovascular disorders and coronary artery diseases. It has been reported that the treatment of anemia by EPO to be associated with improvements in insulin secretion and lipid abnormalities in uremic patients [20,21]. Also, the co-treatment with calcitriol and EPO decreased lipid profiles in this study. Thus, combination therapy with an improved lipid profile and the reduction in total cholesterol and triglyceride can prevent atherosclerosis and cardiovascular disorders. The accumulation of fatty deposits within the lining of an artery is termed atherosclerosis, and atherosclerosis of coronary vessels is a primary cause of heart disease.

The obtained findings demonstrated the increased myocardial injury markers, including creatine kinase-MB and lactate dehydrogenase in the IR group. In contrast, in the group treated with calcitriol, there was a noteworthy decrease in serum LDH, and CK-MB levels and calcitriol restrained the assayed parameters. Recent studies reported that the reduced levels of myocardial injury parameters by the administration of calcitriol in diabetic rats are related to its antioxidant properties [22]. In addition, erythropoietin noticeably reduced the levels of these enzymes. This finding may be due to the antioxidant and anti-inflammatory actions of EPO.

Consistent with this finding, Gholamzadeh et al. [23] indicated the role of EPO in the protection of the heart from ischemic injury and cardiac dysfunction, which decreased the serum levels of LDH and CK-MB after the myocardial infarction and the size of the infarct. Also, in this study, the combination of erythropoietin and calcitriol reduced the serum levels of these enzymes. This indicates the possible protection of the two agents against ischemic injury.

Our histological evaluation showed that ischemia-reperfusion caused glomerular and tubular changes, as shown by acute tubular necrosis, cell detachment, glomerular atrophy, and mononuclear cell infiltration. Sezgin et al. [16] reported that calcitriol has protective effects on IR-induced renal injury, and the morphological changes are reversed by calcitriol treatment. Also, they proposed that calcitriol appears to play a cytoprotective role in the kidney insulted by ischemia-reperfusion. Supporting this proposal, we have found that calcitriol has protective effects on tubular function. This cytoprotective effect of calcitriol may be due to its powerful antioxidant properties. EPO treatment attenuated the pathological changes associated with renal IR injury. Moreover, the attenuating effect of EPO on the morphological changes in renal tissue caused by IR injury has been reported [24]. Also, calcitriol and EPO combination treatment reduced the pathological changes in renal tissue caused by IR injury. The co-treatment (calcitriol +EPO) severely attenuated these changes; nearly the normal renal tissue structure was preserved by calcitriol +EPO pretreatment. Therefore, combination therapy appears to have synergistic protective effects.

In addition, renal IR caused pathological alterations in the cardiac tissue, including intercellular edema, lymphocyte infiltration, and wavy fibers. It seems that the high oxidative stress power of renal IR injury is the main reason for these disorders [1]. We found that the effect of single applications of calcitriol or EPO and the combination of the two agents reduced the morphological changes of the cardiac tissue. These cardio-protective effects of calcitriol 
and EPO may be due to their potent anti-apoptotic, anti-inflammatory, and antioxidant properties, which was documented by the reduction of pathological alterations in the kidney and cardiac tissues. However, the combination treatment was the most effective compared with the other treatment groups.

\section{Conclusions}

In conclusion, cardiac abnormalities and electrical dysfunction are the most important complications after renal ischemia. Calcitriol and EPO have significant anti-inflammatory and antioxidant actions and are well-known free radical scavengers. Our findings demonstrated that the administration of calcitriol or EPO appears to exert a beneficial role on IR- induced cardiac and renal damage, as shown by the decrease of pathological changes and cardiac dysfunction. The treatment with calcitriol and EPO decreased myocardial injury markers and the elevation of lipid profiles- induced by IR. However, the combination treatment with calcitriol and EPO exerted more cardio-protective effects on the single application of them. The present study may serve as a template for further studies on the exact mechanisms mediating the curative effect of calcitriol and EPO combination therapy in IR injury.

\section{Funding}

This study was financially supported by Ardabil University of Medical Sciences, grant number 1883.

\section{Acknowledgments}

Mohammad-Ghasem Golmohammadi: study design and statistical analysis; Ali Shahbazi: English writing and grammar correction; Mir-Mehdi Chinifroush-Asl: histological evaluation; Shokofeh Banaei: study design and biochemical analysis and manuscript writing. All authors read and approved the final manuscript.

\section{Conflicts of Interest}

The authors report no conflicts of interest.

\section{References}

1. Trentin-Sonoda, M.; da Silva, R.C.; Kmit, F.V.; Abrahão, M.V.; Monnerat Cahli, G.; Brasil, G.V.; MuziFilho, H.; Silva, P.A.; Tovar-Moll, F.F.; Vieyra, A.; Medei, E.; Carneiro-Ramos, M.S. Knockout of TollLike Receptors 2 and 4 Prevents Renal Ischemia-Reperfusion-Induced Cardiac Hypertrophy in Mice. PLoS One 2015, 10, e0139350, https://doi.org/10.1371/journal.pone.0139350.

2. Di Lullo, L.; Bellasi, A.; Russo, D.; Cozzolino, M.; Ronco, C. Cardiorenal acute kidney injury: Epidemiology, presentation, causes, pathophysiology and treatment. International journal of cardiology 2017, 227, 143-150, https://doi.org/10.1016/j.ijcard.2016.11.156.

3. Alarcon, M.M.L.; Trentin-Sonoda, M.; Panico, K.; Schleier, Y.; Duque, T.; Moreno-Loaiza, O.; de Yurre, A.R.; Ferreira, F.; Caio-Silva, W.; Coury, P.R.; Paiva, C.N.; Medei, E.; Carneiro-Ramos, M.S. Cardiac arrhythmias after renal I/R depend on IL-1beta. Journal of molecular and cellular cardiology 2019, 131, 101-111, https://doi.org/10.1016/j.yjmcc.2019.04.025.

4. Marcassi, A.P.; Yasbek, D.C.; Pestana, J.O.; Fachini, F.C.; De Lira Filho, E.B.; Cassiolato, J.L.; Canziani, M.E. Ventricular arrhythmia in incident kidney transplant recipients: prevalence and associated factors. Transplant international : official journal of the European Society for Organ Transplantation 2011, 24, $67-$ 72, https://doi.org/10.1111/j.1432-2277.2010.01149.x.

5. Sadeghian, N.; Shadman, J.; Moradi, A.; Ghasem Golmohammadi, M.; Panahpour, H. Calcitriol protects the Blood-Brain Barrier integrity against ischemic stroke and reduces vasogenic brain edema via antioxidant and anti-apoptotic actions in rats. Brain research bulletin 2019, 150, 281-289, https://doi.org/10.1016/j.brainresbull.2019.06.010. 
6. Sinanoglu, O.; Sezgin, G.; Ozturk, G.; Tuncdemir, M.; Guney, S.; Aksungar, F.B.; Yener, N. Melatonin with 1,25-dihydroxyvitamin D3 protects against apoptotic ischemia-reperfusion injury in the rat kidney. Renal failure 2012, 34, 1021-1026, https://doi.org/10.3109/0886022x.2012.700887.

7. Banaei, S.; Ahmadiasl, N.; Alihemmati, A. Combination Anti-Apoptotic Effect of Erythropoietin and Melatonin on Ischemia Reperfusion-Induced Renal Injury in Rats. Acta medica Iranica 2016, 54, 624-630.

8. Banaei, S.; Ahmadiasl, N.; Alihemmati, A. Comparison of the Protective Effects of Erythropoietin and Melatonin on Renal Ischemia-Reperfusion Injury. Trauma monthly 2016, 21.

9. Moore, E.; Bellomo, R. Erythropoietin (EPO) in acute kidney injury. Annals of intensive care 2011, 1, 3, https://doi.org/10.1186/2110-5820-1-3.

10. Hafez, A.A.; Naserzadeh, P.; Ashtari, K.; Mortazavian, A.M.; Salimi, A. Protection of manganese oxide nanoparticles-induced liver and kidney damage by vitamin D. Regulatory toxicology and pharmacology : RTP 2018, 98, 240-244, https://doi.org/10.1016/j.yrtph.2018.08.005.

11. Warnick, G.R.; Knopp, R.H.; Fitzpatrick, V.; Branson, L. Estimating low-density lipoprotein cholesterol by the Friedewald equation is adequate for classifying patients on the basis of nationally recommended cutpoints. Clinical chemistry 1990, 36, 15-19.

12. Kiris, I.; Kapan, S.; Kilbas, A.; Yilmaz, N.; Altuntas, I.; Karahan, N.; Okutan, H. The protective effect of erythropoietin on renal injury induced by abdominal aortic-ischemia-reperfusion in rats. The Journal of surgical research 2008, 149, 206-213, https://doi.org/10.1016/j.jss.2007.12.752.

13. Lai, Y.; Deng, J.; Wang, M.; Wang, M.; Zhou, L.; Meng, G.; Zhou, Z.; Wang, Y.; Guo, F.; Yin, M.; Zhou, $\mathrm{X}$; Jiang, H. Vagus nerve stimulation protects against acute liver injury induced by renal ischemia reperfusion via antioxidant stress and anti-inflammation. Biomedicine \& pharmacotherapy 2019, 117, https://doi.org/10.1016/j.biopha.2019.109062.

14. Alzahrani, F.A. Melatonin improves therapeutic potential of mesenchymal stem cells-derived exosomes against renal ischemia-reperfusion injury in rats. American journal of translational research 2019, 11, 28872907.

15. Ahmadiasl, N.; Banaei, S.; Alihemmati, A.; Baradaran, B.; Azimian, E. The anti-inflammatory effect of erythropoietin and melatonin on renal ischemia reperfusion injury in male rats. Advanced pharmaceutical bulletin 2014, 4, 49-54, https://doi.org/10.5681/apb.2014.008.

16. Sezgin, G.; Ozturk, G.; Guney, S.; Sinanoglu, O.; Tuncdemir, M. Protective effect of melatonin and 1,25dihydroxyvitamin D3 on renal ischemia-reperfusion injury in rats. Renal failure 2013, 35, 374-379, https://doi.org/10.3109/0886022x.2012.760409.

17. Bonakdaran, S.; Ayatollahi, H.; Mojahedi, M.J.; Sharifipoor, F.; Shakeri, M. Impact of treatment with oral calcitriol on glucose intolerance and dyslipidemia(s) in hemodialysis patients. Saudi journal of kidney diseases and transplantation : an official publication of the Saudi Center for Organ Transplantation, Saudi Arabia 2008, 19, 942-947.

18. Eftekhari, M.H.; Akbarzadeh, M.; Dabbaghmanesh, M.H.; Hassanzadeh, J. The effect of calcitriol on lipid profile and oxidative stress in hyperlipidemic patients with type 2 diabetes mellitus. ARYA atherosclerosis 2014, 10, 82-88.

19. Tuzcu, A.; Bahceci, M.; Yilmaz, E.; Bahceci, S.; Tuzcu, S. The comparison of insulin sensitivity in nondiabetic hemodialysis patients treated with and without recombinant human erythropoietin. Hormone and metabolic research 2004, 36, 716-720, https://doi.org/10.1055/s-2004-826021.

20. Pollock, C.A.; Wyndham, R.; Collett, P.V.; Elder, G.; Field, M.J.; Kalowski, S.; Lawrence, J.R.; Waugh, D.A.; George, C.R. Effects of erythropoietin therapy on the lipid profile in end-stage renal failure. Kidney international 1994, 45, 897-902, https://doi.org/10.1038/ki.1994.118.

21. Khedr, E.; El-Sharkawy, M.; Abdulwahab, S.; Eldin, E.N.; Ali, M.; Youssif, A.; Ahmed, B. Effect of recombinant human erythropoietin on insulin resistance in hemodialysis patients. Hemodialysis international. International Symposium on Home Hemodialysis 2009, 13, 340-346, https://doi.org/10.1111/j.1542-4758.2009.00367.x.

22. El-Mansi, A.A.; Al-Kahtani, M.A. Calcitriol and Punica Granatum Extract Concomitantly Attenuate Cardiomyopathy of Diabetic Mother Rats and Their Neonates via Activation of Raf/MEK/ERK Signalling and Mitigation of Apoptotic Pathways. Folia biologica 2019, 65, 70-87.

23. Gholamzadeh, A.; Amini, S.; Mohammadpour, A.H.; Vahabzadeh, M.; Fazelifar, A.F.; Fazlinezhad, A.; Dehghani, M.; Moohebati, M.; Dastani, M.; Malaekeh-Nikouie, B.; Falsoleiman, H. Erythropoietin Reduces Post-PCI Arrhythmias in Patients With ST-elevation Myocardial Infarction. Journal of cardiovascular pharmacology 2015, 65, 555-561, https://doi.org/10.1097/fjc.0000000000000223.

24. Ahmadiasl, N.; Banaei, S.; Alihemmati, A. Combination antioxidant effect of erythropoietin and melatonin on renal ischemia-reperfusion injury in rats. Iranian journal of basic medical sciences 2013, 16, 1209-1216. 